\title{
Investigation of Possible Failure Locations: KDE of Intersection Points on Visible Rock Surfaces
}

\author{
Alparslan Turanboy ${ }^{1}$, Erkan Ülker $^{2}$, Cahit B. Küçüksütcü ${ }^{3}$ \\ ${ }^{1}$ NE University, Department of Mining Engineering, Anabağlar District No 11, Konya, Turkey \\ ${ }^{2}$ Selçuk University, Department of Computer Engineering, Ardıçlı District, Konya, Turkey \\ aturanboy@konya.edu.tr, eulker@selcuk.edu.tr \\ ${ }^{3}$ Department of Software Engineering, BayE Information, Education and Consulting Ltd., \\ Akademi District Gürbulut St. No 67 F-116 Konya, Turkey \\ cbkucuksutcu@bayebilisim.com
}

\begin{abstract}
One of the most serious problems in rock engineering applications is the accidental falling of rock blocks that are formed by discontinuity in the rock mass and free surface(s) of engineering structure such as open pit slope, tunnel wall etc. For the stability analysis of in rock mass, there are so many parameters. However, the most important of them which should be taken into account, are the geometrical parameters of discontinuity because the discontinuities are mechanically weak zones and failures occurred on thought surfaces and intersection lines of them and geometrical characteristics are also used as decisive factors in different scales in all stability analysis. Investigation of starting point of possible fails must be a crucial step both in pre-design analysis on existing structures. Because the failures are mostly beginning at these points which are investigated as an analytic solution in this paper. Starting possible locations of failures can be investigated as points on free surfaces of an engineering construction. This study includes derived series of linear equations for the investigation of these points. In addition, Kernel Densities Estimation (KDE) of intersection points is described using for the prediction of failure locations on the visible surfaces of an engineering structure. Derived methodology was sampled on an experimental highway wall and consistent results were shown successfully.
\end{abstract}

Keywords - rock mass, discontinuity, kernel densities estimation, stability

\section{Introduction}

The natural or artificial rock slopes in areas where people live and use, have engendered stability related problems caused mainly without regarding for environmental factors and inadequate plannings and design studies (Figure 1).Several researchers, who studied this topic, have described the evaluation of discontinuity data. For example, plotting discontinuity data on stereograms, contouring to determine prominent orientations and subsequent wedge analyses are described in rock slope engineering [1] methods of geological engineering in discontinuous rock and introduction to rock mechanics [2].

Wedge failure is the most common failure mode that is structurally controlled by two oblique discontinuities. Potential sliding of a tetrahedron form occurs along the day-lighted intersection line. To be kinematicaly viable, such a wedge must have an intersection line that falls between the dip directions of the two planes, and must be parallel (or nearly parallel) to the dip direction of the slope face. Various investigators have previously explained wedge failure analyses elaborately. The basic limiting equilibrium method for wedge failure was represented by [3] and detailed for different conditions by [4], as well as [1]. When the downward movements of a rock block due to gravity and shear stresses exceed the shear strength, failure occurs. In situ rock blocks that may fail are formed by intersecting discontinuities and the free face created through excavation of an opening. In addition, the geometry, size, and spatial position of any rock block have an effect on whether failure will happen or not, failure direction, and which failure modes may occur. It is broadly assumed that at that place are three fundamental failure modes in rock slopes: wedge, planer, and toppling failures. Figure 2 illustrates what is traditionally regarded as these three basic failure modes [5].

Discontinuity parameters can be classed as either geometrical or non-geometrical. Non-geometrical properties which related to mechanical properties of intact rock, while geometrical properties define as the fabric of the rock mass [6]. The geometrical parameters, which are dip, dip direction, orientation, spacing, and length of discontinuity, have been extensively explained by [7]. These data are directly gathered from rock mass exposure or bore holes in the rock mass, and are generally used to directly understand the statistical nature of discontinuities and the detailed instability analyses. 


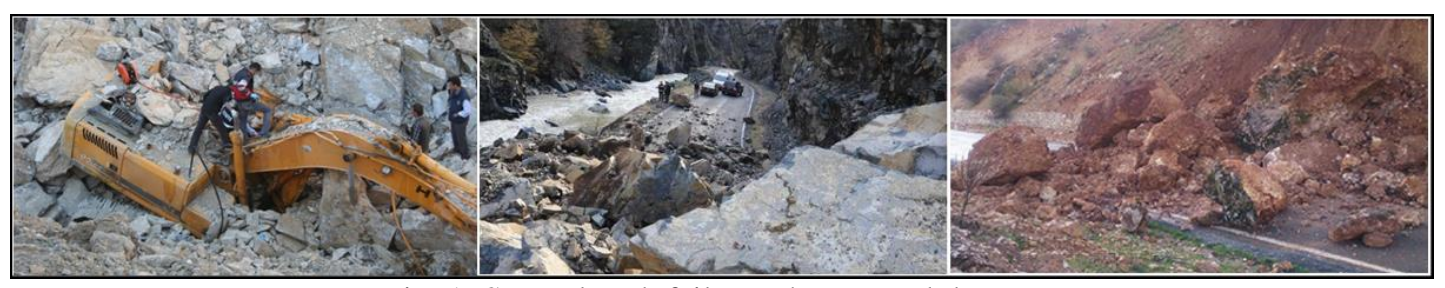

Fig. 1: Several rock failures that caused damage.

For example, plotting and contouring the data on a stereogram are being used to determine prominent orientations for the scope of classical kinematic analyses. On the other hand, the directly measured parameters are also being used to derive quasi-physical parameters about the size and shape of the rock blocks. In addition, volume coefficient density and weighted joint density are being extensively studied by several researchers [8], [9], [2], [10].

This study is motivated by the need to develop an improved way to predict rock failures in rock slopes. Slope stability estimation is an important engineering problem that involves several parameters which should be investigated and interpreted carefully on, especially, artificial slopes. The prior parameters that should be examined ones are the geometry and orientation features of slope and discontinuities (fault, joint, bedding, etc.) in any engineering application related to excavation in a rock mass. In preliminary and detail design studies, stability of rock slopes are often numerically analyzeddepends on several complex techniques with using deterministic and/or statistic processes.

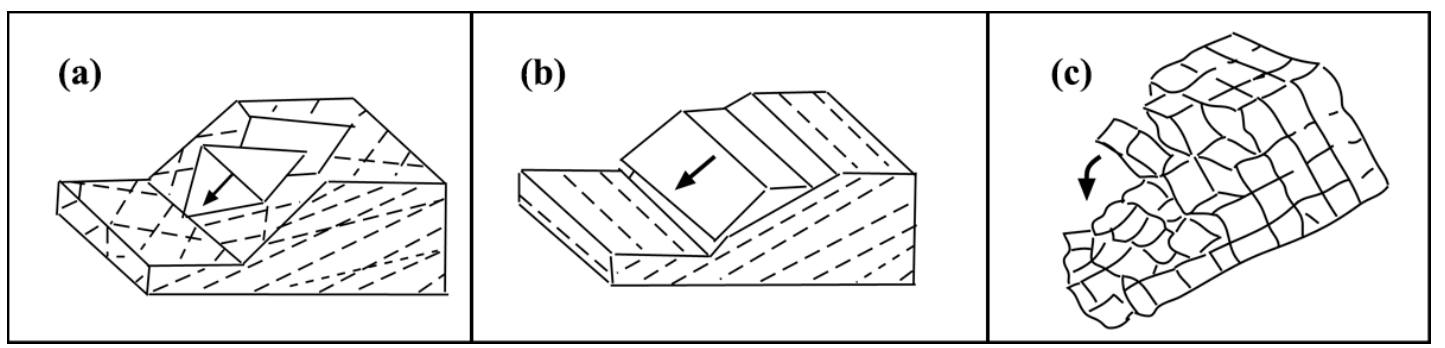

Fig. 2. Three failure modes in rock slopes; a) wedge, b) planer and c) toppling failures (from Matheson, 1983).

These techniques have been used successfully in a wide range of rock engineering applications but often both expensive and time-consuming tasks, especially, in the first assessments. In this study, a visual presentation approach to predict the critical failure zones on the visible surfaces of rock slopes has been suggested. The approach that suggested in this study is based on exploratory data analysis. Primary and derived data have been used in the developed approach as the main outputs. Results that obtained from this approach can be an indicator for several instabilities.

The intersection points of failure were investigated using scatter and densities of intersection points on free surfaces of a rock slope. Kernel Density Estimation (KDE) has been used as a main tool for statistical investigation of critical instability locations. KDE is a popular tool for $2 \mathrm{D}$ visualizing the distribution of scattered data among numerous engineering aims because it is easy to interpret. The $2 \mathrm{D}$ kernel density plot is a smoothed colour density representation of the scatterplot is based on kernel density estimation a nonparametric technique for probability density functions [11] This tool has ever-expanding applications in several visualization aims, and it seems to be a robust analysis in Geotechnical applications, as it supports geospatial data analysis.

In addition, the basic survey method, called a scanline sampling, was used in this study. Scanline surveys consist of directional and qualitative measurements of rock discontinuities [12]. This survey method is widely used in geology and engineering investigations of fractured rock masses.

\section{Methodology}

In this analytic study, 2D kernel density contouring of intersection points (can be regarded as evaluated or derived data) are suggested as a tool for the investigation and prediction accordingly the failure locations on the visible surfaces of an engineering structure. The logical order of the model can be ordered as;

Collection of relevant data on the exposure using scanline technique $\rightarrow$ deriving coordinates of intersection points (derived data) $\rightarrow 2 \mathrm{D}$ modelling of scatter points and kernel density distribution of intersection points on the free rock mass surfaces $\rightarrow$ interpretation of results regarded instabilities.

The first part of the modelling study includes the construction of the trace lines geometry which belongs to possible rock blocks on the free surfaces of rock slopes. 
Excavation surface and upper slope surface have been examined as three parts in the 2D visualizations. Here, excavation surface was divided into two parts (areas I and II) by a line from the intersection point $\left(I_{l}\right)$, though y axis and upper slope are considered to be one area (area III). When the visible traces on areas I and II are considered into account, all formed intersection points can be identified easily. (Figure 3)

Fundamentals of the model were calculated based on the intersection points of two line segments from their parametric form in a 3D Cartesian coordinate system, and they were then connected systematically. Given points $\mathrm{P}_{1}=$ $\left(\mathrm{x}_{1}, \mathrm{y}_{1}, \mathrm{z}_{1}\right)$ and $\mathrm{P}_{2}=\left(\mathrm{x}_{2}, \mathrm{y}_{2}, \mathrm{z}_{2}\right)$, the parametric forms for the two lines are given in equation 1 . The intersection point of two lines can be determined by using the simultaneous equation method: In these equations, (and it is linear coefficients $(0 \leq \lambda \leq 1$ and $0 \leq \mathrm{t} \leq 1)$ and $\alpha, \beta$, and (are clockwise angles to the $\mathrm{x}, \mathrm{y}$, and $\mathrm{z}$ axes then the intersection point of the two lines is solved as $\left(\mathrm{x}_{3}, \mathrm{y}_{3}, \mathrm{z} 3\right)$.

$$
\begin{aligned}
& x_{1}+\lambda \cos \alpha_{1}=x_{2} t \cos a_{2}=x_{3} \\
& y_{1}+\lambda \cos \alpha_{1}=x_{2} t \cos a_{2}=y_{3} \\
& z_{1}+\lambda \cos \alpha_{1}=x_{2} t \cos a_{2}=z_{3}
\end{aligned}
$$

For the 2D explanation, the intersection point $\left(I_{2}\right)$ on $\mathrm{XY}$ was transformed to the $\mathrm{YZ}$ plane using a rotation matrix (equation 2).

$$
\left(x^{\prime} y^{\prime}\right)=(x y)\left[\begin{array}{cc}
\cos 90^{\circ} & \sin 90^{\circ} \\
-\sin 90^{\circ} & \cos 90^{\circ}
\end{array}\right]
$$

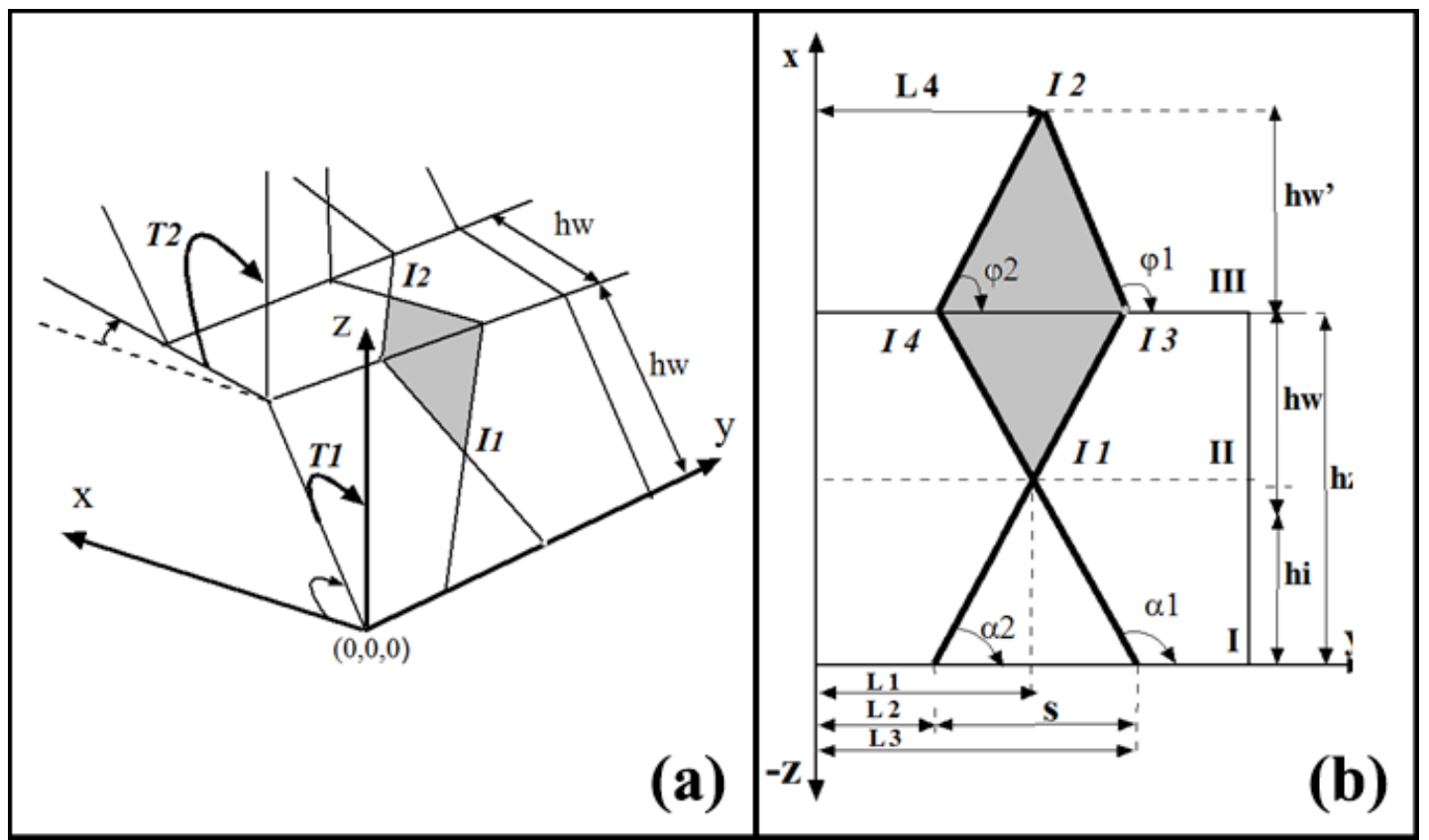

Fig. 3: A typical wedge mode that formed two diagonal discontinuity traces; on a) 3D slope (T1 and T2 represent first and second transform), b) 2D transformed the visible surface of basic wedge and dimensions.

Two data types have been used for construction of KDE distribution of the intersection points; the first data type has been recorded from an outcrop of rock mass directly by scanline surveying and secondary data have been derived from the first data. The second data consists of only intersection points on slope faces. Intersection points have been derived from a series of geometric and empirical equations.

\section{Field Experiment}

The study area consists of an extensive rock cut along the Konya-Antalya Highway in southern Turkey, near the Seydişehir (Figure 4). This area along this highway shows exceptional exposure of series of limestone formations. Discontinuity data have been obtained by the authors from a field survey on a road cut $20 \mathrm{~km}$ from Seydişehir. The length of sampled road cut is approximately $30 \mathrm{~m}$ and comprises one slope. During the fracture survey, 33 discontinuity values were measured on the excavated surface of sampled rock cut. 


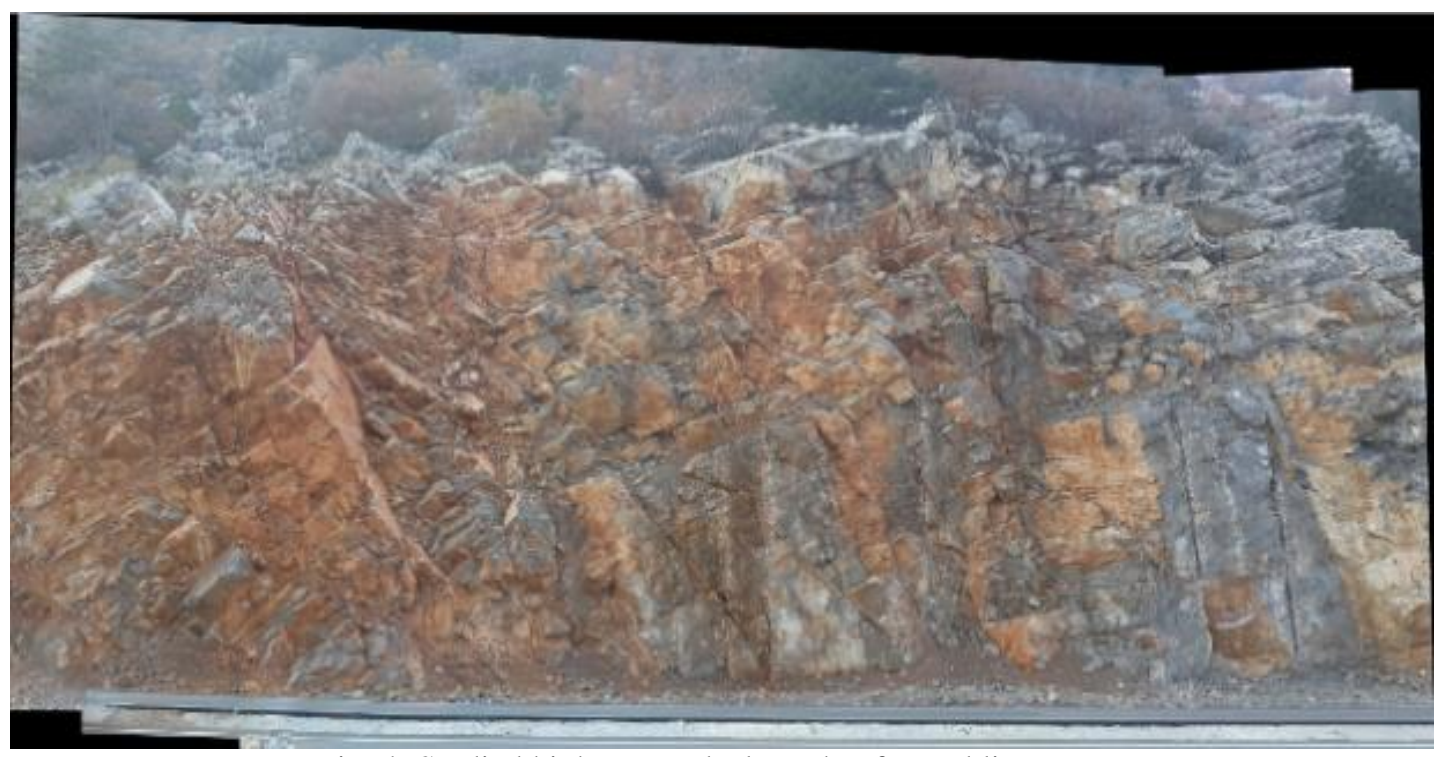

Fig. 4: Studied highway rock slope that formed limestone.

Represented prism dimensions were selected as $15 \times 30 \times 12 \mathrm{~m}$, and 33 data were recorded along the $30 \mathrm{~m}$ scanline. The important assumption is that any discontinuity is neighter vertical nor horizontal to each other. Thence, 528 intersection points can be estimated by using general combination equation (equation 3$)((33 ! / 2 !(33-2) !)=528)$ in space. On the other hand, 73 and 183 intersection points were calculated on surfaces XY and YZ, respectively, from the 33 recorded data using equations 1 and 2 . This means that approximately $46 \%$ of all intersection points $(183+73 / 528) \times 100$ $\approx 46 \%$ ) were modelled on the XY and YZ surface boundaries.

$$
C(n, r)=n ! /[r !(n-r) !]
$$

Kernel Density Estimation (KDE) is used in statistics to estimate probabilities of the density function of random variable. KDE is commonly being used to smooth densities based on a finite data set. A kernel density estimation can be defined as in the equation 4 for a $1 D$ data. $K$ is a function which is satisfying $\int K(x) d x=1$ and the function $K$ is referred to as the kernel. $\mathrm{h}$ is a positive number, usually called the bandwidth.

$$
f_{h}(x)=\frac{1}{n} \sum_{i=1}^{n} K_{h}\left(x-x_{i}\right)=\frac{1}{n h} \sum_{i=1}^{n} K\left(\frac{x-x_{i}}{h}\right)
$$

In the statistical part of the study, a Matlab code has been developed which applies KDE for the intersection points on free surfaces of rock slope. Matlab R2014b has been used for the purpose of point data contouring in the study. An intersection line defined by points $\mathrm{P}_{0}$ and $\mathrm{P}_{1}$ and surface defined with point $\mathrm{V}$ and normal $\mathrm{n}$; intersection point for surface and intersection line can be calculated (equation $5 \mathrm{a}, \mathrm{b}$ and c) using a derived linear equation (a) where $\mathrm{u}$ is the difference vector of $\mathrm{P}_{1}$ and $\mathrm{P}_{0}(\mathrm{~b})$ and $\mathrm{w}$ is the difference vector of $\mathrm{P}_{0}$ and $\mathrm{V}(\mathrm{c})$.

$$
\begin{gathered}
P=P_{0}+(-(n \cdot w)) /(n \cdot u) u \\
u=P_{1}-P_{0} \\
w=P_{0}-V
\end{gathered}
$$

There are various kernel functions; uniform, triangle, epanechnikov, quartic, triweight, gaussian, cosiness etc. Gaussian kernels are commonly used in several data visualization applications. It uses with appropriate regularization guarantees a globally optimal predictor that has minimum approximation and estimation errors. Here, the approximation error refers to the error which incurred by limiting the space of classification models over which search is performed and estimation error refers to an error in the estimation of the model parameters. In addition, it is also convenient because of its linear and polynomial character. The Gaussian kernel function is used to calculate densities where $t$ is the matrix contains a 2D vector list of intersection points (equation 6). 


$$
K(u)=\frac{1}{\sqrt{2 \pi}} \exp \left(-\frac{1}{2} u^{2}\right)
$$

In KDE applications, bandwidth has the key importance in the estimations. Taking the bandwidth too large or too small, lead to false estimation and overstatements. Too small values of bandwidth lead to smoothing loss and spiky estimation while too large values of bandwidth leads to loosing data and too much smoothing. Considering these requirements, bandwidths have been chosen as three alternatives.

Kernel density contours of the derived intersection points and scattered intersection point data on surfaces XZ and YZ are shown in Figure 5 respectively.

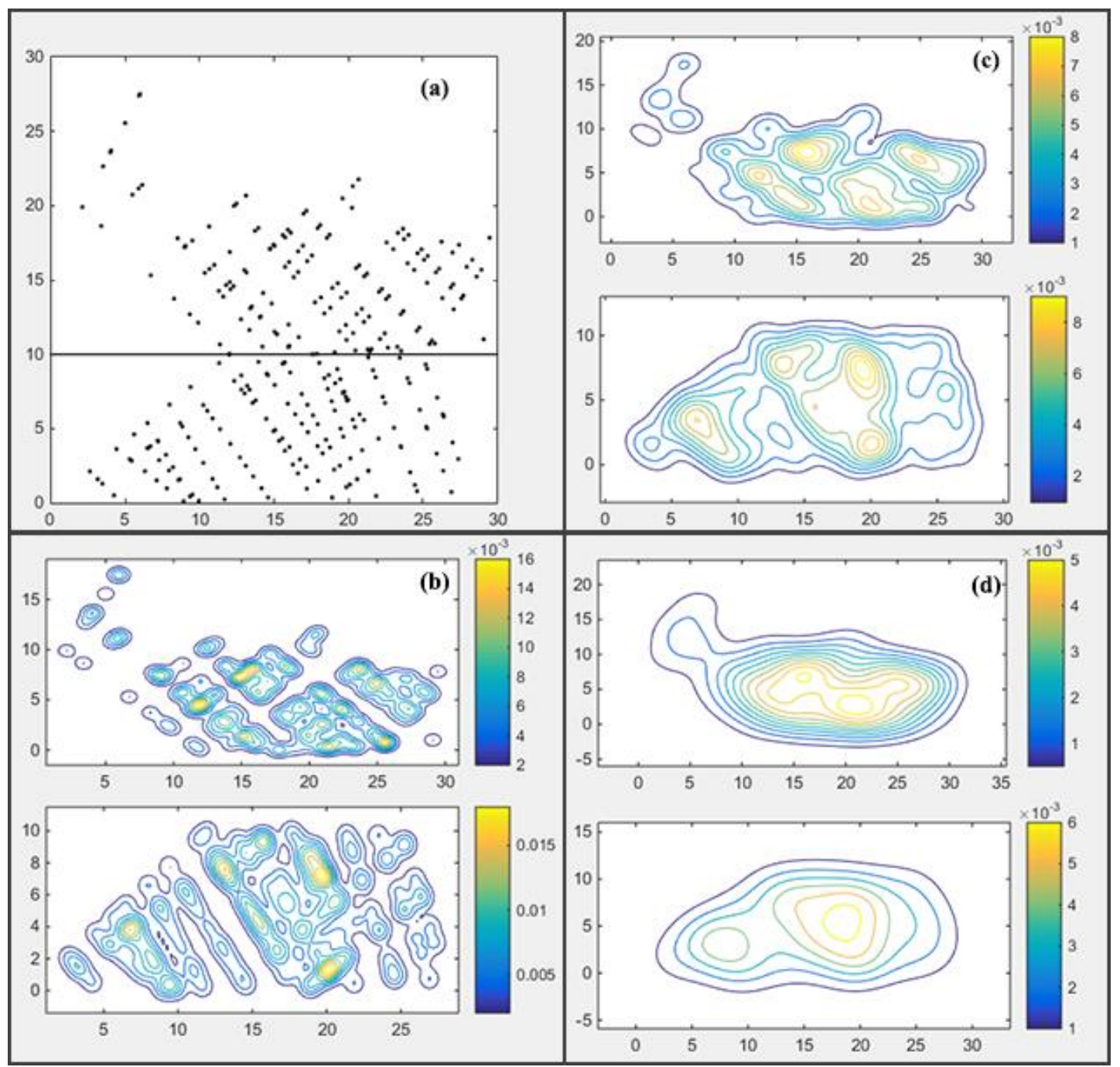

Fig. 5: a) Scatter plot and Bivariate Kernel Densities for three bandwidths; b) $0,5 \mathrm{~m} \times 0,5 \mathrm{~m}$, c) $1,0 \mathrm{~m} \times 1,0 \mathrm{~m}, \mathrm{~d}) 2,0 \mathrm{~m} \times 2,0 \mathrm{~m}$.

\section{Conclusion}

Kernel density estimation is an effective way to demonstrate cluster data. It is used across the statistical spectrum to fit smoothed boundaries to observed data. Using KDE with point plots together have enabled to see singular weak points while interpreting the overall weak zones. Results have been obtained that are more general, while the selection from small sized to large sized bandwidth. Undoubtedly, the zones where concentrations of intersection points increased are mechanically weaker. It is possible to represent the complexity of rock structure and prediction of failed zones using modelling of these weak zones.

It can be clearly follown that intersection zones belonging to bedding and joints for the first banwitdh selection. The other two selections give more general results and general instabilities are approximately located in the middle of modelled frames. While bandwidth increases, the model will give us more general results. 
The model enables us to make the first and faster evaluations of stability problems on visible, inaccessible and invisible surfaces, which became degraded because of weathering and vegetation cover

In addition, the model can be adapted to other detailed surveying techniques such as photo analyses, because all recording data used can be transformed to the Cartesian coordinate system.

\section{References}

[1] E. Hoek ve and J. W. Bray, Rock Slope Engineering, Revised 3rd Edition. London: The Institution of Mining and Metallurgy, 1981.

[2] R. E. Goodman, Introduction to Rock Mechanics. New York: John Wiley \& Sons, 1989.

[3] W. Wittke, "Influence of the Shear Strength of the Joints on the design of Pre-stressed Anchors to stabilize a Rock Slope," in \%1 içinde Geotechnical Conference, Oslo, 1967.

[4] K. Kovaâri ve and P. Fritz, "Stability Analysis of Rock Slopes for Plane and Wedge Failure with the Aid of a Programmable Pocket Calculator," in \%1 içinde 16th US Rock Mechanics Symposium, Minneapolis, 1975.

[5] G. D. Matheson, "Rock Stability Assessment in Preliminary Site Investigations Graphical Methods," Crowthorne, Berkshire: Transport and Road Research Laborator, 1983.

[6] R. H. Hack, "Slope Stability Probability Classification 2nd Edition," \%1 içinde SSPC ITC, Enschede, 1998.

[7] ISRM, "Suggested Methods for the Quantitive Description of Discontinuities," International Society of Rock Mechanics, pp. 319-368, 1981.

[8] ISRM, Rock Characterization, Testing and Monitoring ISRM Suggested Methods. Oxford: Pergamon, 1981.

[9] H. Wang, J. P. Latham ve, and A. B. Poole, "Predictions of Block Size Distribution for Quarrying," Quarterly Journal of Engineering Geology, pp. 91-99, 1991.

[10] A. Palmström, "Recent Developments in Rock Support Estimates by the RMi," Hournal of Rock Mechanics and Tunnelling Technology, pp. 1-19, 2000.

[11] D. Lucy, R. G. Aykroyd ve, and A. M. Pollard, "Non-Parametric Calibration for Age Estimation," Applied Statistics, pp. 183-196, 2002.

[12] S. D. Priest ve and J. A. Hudson, "Estimation of Discontinuity Spacing and Trace Length Using Scanline Survey," International Journal of Rock Mechanics and Mining Sciences, pp. 183-197, 1981. 